\title{
Admission triage tool for adult intensive care unit admission in Hong Kong during the COVID-19 outbreak
}

\author{
Gavin M Joynt *, Anne KH Leung, CM Ho, Dominic So, HP Shum, FL Chow, Alwin WT Yeung, KL Lee, \\ Gloria KY Tang, WW Yan; for the Triage Working Group of the Co-ordinating Committee (Intensive Care)
}

\begin{abstract}
A B S T R A C T
Intensive care is expensive, and the numbers of intensive care unit (ICU) beds and trained specialist medical staff able to provide services in Hong Kong are limited. The most recent increase in coronavirus disease 2019 (COVID-19) infections over July to August 2020 resulted in more than 100 new cases per day for a prolonged period. The increased numbers of critically ill patients requiring ICU admission posed a capacity challenge to ICUs across the territory, and it may be reasonably anticipated that should a substantially larger outbreak occur, ICU services will be overwhelmed. Therefore, a transparent and fair prioritisation process for decisions regarding patient ICU admission is urgently required. This triage tool is built on the foundation of the existing guidelines and framework for admission, discharge, and triage that inform routine clinical practice in Hospital Authority ICUs, with the aim of achieving the greatest benefit for the greatest number of patients from the available ICU resources. This COVID-19 Crisis Triage Tool is expected to provide structured guidance to frontline doctors on how to make triage decisions should ICU resources become overwhelmed by patients requiring ICU care, particularly during the current COVID-19 pandemic. The triage tool takes the form of a detailed decision aid algorithm based on a combination of established prognostic scores, and it should increase objectivity and transparency

in triage decision making and enhance decisionmaking consistency between doctors within and

across ICUs in Hong Kong. However, it remains an aid rather than a complete substitute for the carefully considered judgement of an experienced intensive care clinician.

Hong Kong Med J 2022;28:64-72

https://doi.org/10.12809/hkmj209033

${ }^{1}$ GM Joynt *, MBBCh, FHKAM (Anaesthesiology)

${ }^{2}$ AKH Leung, MB, ChB, FHKAM (Anaesthesiology)

${ }^{3} \mathrm{CM}$ Ho, MB, ChB, FHKAM (Medicine)

${ }^{4}$ D So, MB, BS, FHKAM (Anaesthesiology)

${ }^{5}$ HP Shum, MB, BS, MD

${ }^{6}$ FL Chow, MB, BS, FHKAM (Medicine)

${ }^{7}$ AWT Yeung, MB, BS, FHKAM (Medicine)

${ }^{8} \mathrm{KL}$ Lee, MB, ChB, FHKAM (Medicine)

${ }^{9}$ GKY Tang, MB, BS, FHKAM (Medicine)

${ }^{5}$ WW Yan, MB, BS, FHKAM (Medicine)

${ }^{1}$ Department of Anaesthesia and Intensive Care, The Chinese University of Hong Kong, Hong Kong

2 Department of Intensive Care, Queen Elizabeth Hospital, Hong Kong

Department of Intensive Care, Tuen Mun Hospital, Hong Kong

Department of Intensive Care, Princess Margaret Hospital, Hong Kong

Department of Intensive Care, Pamela Youde Nethersole Eastern Hospital, Hong Kong

${ }^{6}$ Department of Intensive Care, Caritas Medical Centre, Hong Kong Department of Medicine and Geriatrics, Ruttonjee \& Tang Shiu Kin Hospitals, Hong Kong

${ }^{8}$ Department of Intensive Care, United Christian Hospital, Hong Kong

${ }^{9}$ Department of Adult Intensive Care, Queen Mary Hospital, Hong Kong

* Corresponding author: gavinmjoynt@cuhk.edu.hk
\end{abstract}

This article was published on 28 Jan 2021 at www.hkmj.org.

\section{Introduction}

The most recent wave of coronavirus disease 2019 (COVID-19) infections in July to August 2020 resulted in more than 100 new cases per day in Hong Kong for a prolonged period. The stress experienced by individual intensive care units (ICUs) in Hong Kong was demonstrated by the need for an unusually large number of patient transfers between units to maximise the available ICU capacity, despite the implementation of surge strategies. Admission decisions to ICUs resulting from the added pressure for ICU beds, as well as social dimensions that were triggered by the COVID-19 outbreak, resulted in an urgent requirement for contingencies to inform admission triage practices in the face of overwhelming ICU demand. Professional bodies have recommended that triage protocols (clinical decision support systems), rather than clinical judgement alone, be used in triage whenever possible, ${ }^{1}$ and that such protocols be available to assist frontline doctors. ${ }^{1,2}$ Such protocols should be locally relevant and prepared in advance of the need for implementation.

This document is built on the existing 
Admission, Discharge, and Triage Guidelines that inform routine clinical practice in Hospital Authority ICUs. The purpose of this COVID-19 Crisis Triage Tool is to provide structured guidance to frontline clinicians to assist with triage decision making should ICU resources become overwhelmed by patients requiring ICU care in Hong Kong.

\section{Background}

The Admission, Discharge, and Triage Guidelines for Adult Intensive Care Services for use in dayto-day ICU operations in Hong Kong were recently updated in an internal operations circular in 2018. A brief summary of this guideline follows. Hong Kong ICUs provide a high standard of intensive care by international benchmarks. ${ }^{3}$ However, because of the expensive nature of intensive care resources, there are a limited number of ICU beds available in Hong Kong. Hong Kong has approximately 7.1 critical care beds per 100000 population, a low number compared with other high-income regions in Asia (Singapore: 11.4/100 000, Taiwan: 29/100000), North America (Canada: 12/100000, United States: 20/100000), and Europe (Germany: 25/100000, Belgium: 20/100000). 4,5 Therefore, ICU beds in Hong Kong are generally reserved for patients with reversible medical conditions who have reasonable prospects of substantial recovery, and triage (prioritisation) decisions are routinely necessary. ${ }^{6,7}$ The existing admission and triage guidelines are designed to help optimise the use of ICU services to achieve the largest possible benefit for the most patients within available resources, a modified utilitarian ethical approach that is recommended by ICU professional bodies internationally. ${ }^{8-10}$ Briefly, patients who require ICU care are referred to the ICU team for admission screening. All ICU admission triage decisions are supervised by a senior, experienced ICU doctor and implemented according to individual unit policy. In principle, all triage decisions should be based on the patient's medical condition and the benefits likely to be derived from ICU admission (in comparison with a lower level of care). Non-medical factors such as gender, race, religion, education level, and social status should not be considered when making triage decisions. The existing broad-based framework that guides individual unit policy (Fig 1) was used to inform the relevant components of the new COVID-19 Crisis Triage Tool.

\section{Maximising existing intensive care unit capabilities in response to a surge of COVID-19 cases}

Prioritisation for ICU admission in the form of admission triage can only be justified once all efforts to maximise available resources have been exhausted. The Hospital Authority's existing

\section{香港COVID-19爆發期間的深切治療部住院 分流工具}

Gavin M Joynt、梁結雄、何俊明、蘇㭌頎、沈海平、 㱀富來、楊維德、李家龍、鄧佳欣、殷榮華; 統籌委員會 分流工作小組（深切治療）

在香港, 深切治療可提供的病床和受訓醫護人員數目有限。2020年 7 月至 8 月期間, 2019 冠狀病毒病（COVID-19）曾持續每天新增超 過 100 宗個案。危重患者入住深切治療部的需求增加, 對本地醫療系 統構成挑戰, 預期發生更大規模的爆發時, 深切治療服務將不勝負 荷。因此, 一個透明和公平的深切治療分流是有迫切需要的。這個分 流工具建基於現時的住院、出院和分流指南及框架上, 指南和框架為 醫院管理局深切治療部的常規臨床實踐提供參考, 使深切治療部的資 源能為最多患者帶來最大利益。COVID-19危機分流工具預計可為前 線醫生提供有序指引, 在深切治療部資源不堪重負時（尤其於當前 COVID-19大流行期間 ），對需要深切治療部護理的患者作適當的分 流決策。分流工具採用預後評分, 增加分流決策的客觀性和透明度, 有助本地院內和跨院深切治療部醫生決策的一致性。然而, 這個分流 工具只能擔當輔助角色, 並不能取代深切治療部醫生的臨床判斷。

infectious disease contingency plan dictates that the number of available ICU beds be increased and is based on certain key principles: first, that the standard of intensive care should be maintained at a standard similar to that usually provided by Hospital Authority ICUs, and second, that infection control procedures that provide a high level of protection against staff cross-infection with severe acute respiratory syndrome coronavirus 2 (SARS-CoV-2) should be maintained. Maintaining these standards requires the provision of appropriately trained staff in adequate numbers. Failure to adhere to these principles may have devastating consequences, as occurred during the SARS outbreak in 2003. ${ }^{11}$ The requirement to maintain these standards necessarily results in a relatively limited surge capacity ${ }^{12}$ that may be incapable of meeting all the demands of a large COVID-19 transmission surge in the community. Thus, the overall increase ( 68 beds) in ICU airborne infection isolation room beds from Stage I (48 beds) to Stage III (116 beds) will likely be insufficient.

The Hong Kong Government has plans to construct a number of temporary community hospitals for a potentially large surge. However, this initiative does not include a provision for ICU beds, and independent preparations will be required to maximise ICU capacity. In the event that individual hospitals need to increase ICU capacity beyond that specified by the existing Contingency Plan Stage III provisions, a number of key principles should be adopted (Table 1). 


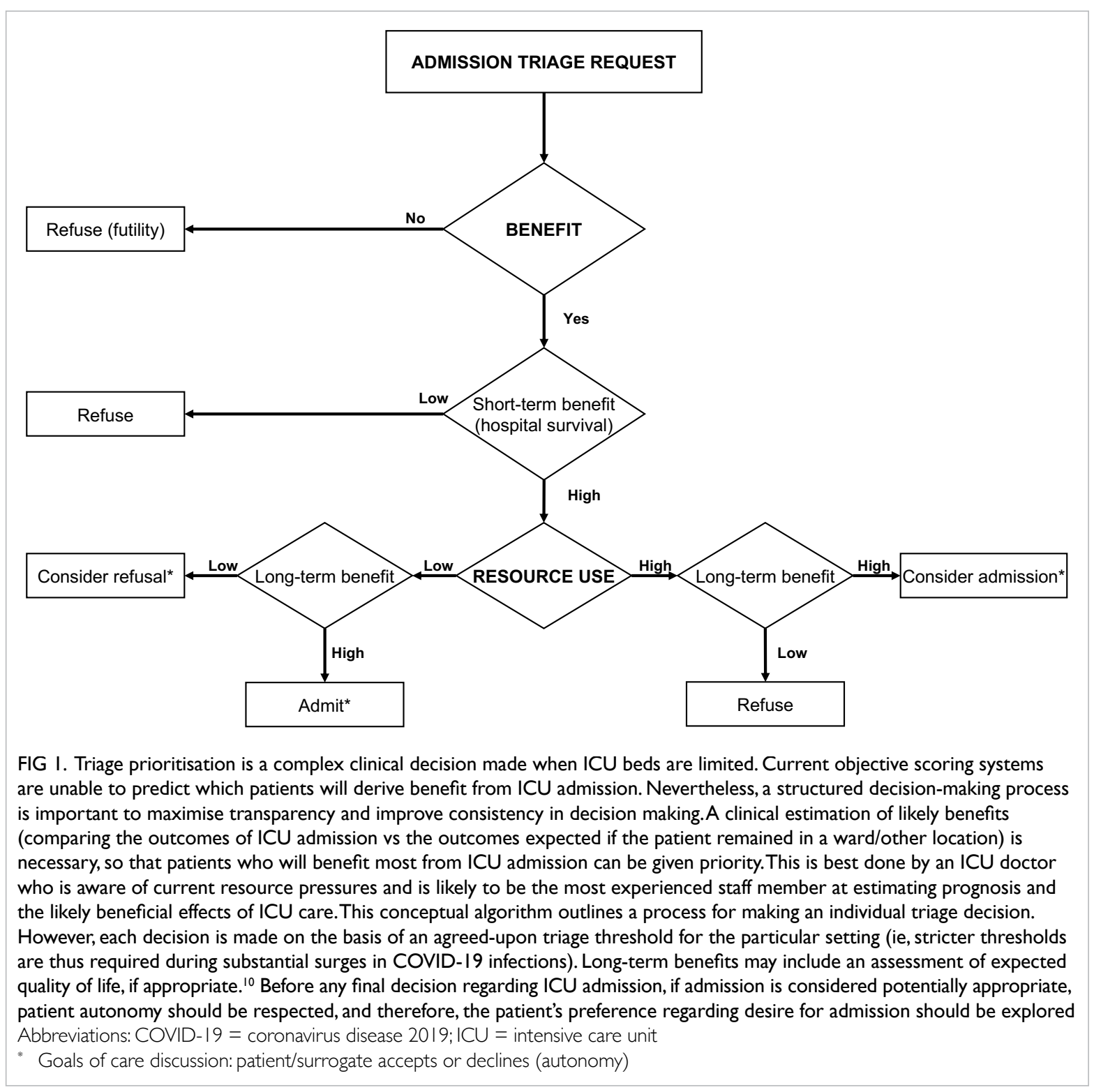

TABLE I. Principles to be adopted for ICU bed provision and triage beyond the Stage III Contingency Plan (Crisis stage)

1. The increase in ICU bed number cannot be solely dependent upon demand for admission or mechanical ventilation but should be determined by the reasonable limits of trained staff and safe facilities.

2. Patient and staff safety should be prioritised and appropriate protection from disease transmission ensured. Thus, the availability of appropriate AIIR facilities for ICU patients will be a key requirement for expansion beyond those available ICU facilities during contingency plan stage III activation.

3. The threshold ratios of ICU specialty staff to non-ICU specialty staff (deployed in from other specialties) and staff to patients should be regularly reviewed and meet current standards set by the HA.

4. The usual triage principles as outlined in the accompanying documents should be adopted during this phase. However, higher thresholds for admission will be required based on prediction of mortality, and such thresholds will be determined by ICU bed availability and the magnitude of the increased demand.

5. Although good communication between specialties caring for individual patients is of great importance, decisions made by the ICU COS/Director shall be final. The hospital ethics committee should provide advice for difficult cases and unresolvable conflicts.

6. The threshold for initiating organ support, especially resource-intensive means of support such as ECMO, should be aligned across HA ICUs and be regularly reviewed with regard to the surge situation.

7. Reliable communication channels and coordination should be maintained on an intra-departmental basis within ICUs and between the ICU, the hospital, and the HA Head Office.

Abbreviations: AlIR = airborne infection isolation rooms; $C O S=$ Chief of Service; ECMO = extracorporeal membrane oxygenation; $\mathrm{HA}=$ Hospital Authority; ICU = intensive care unit 


\section{Methods}

Despite some previous attempts, no single objective, evidence-based triage tool in the form of a score or combination of scores has been shown to effectively determine appropriate ICU admission priority. ${ }^{13-15}$ Development of a guidance tool was thus initiated using an iterative process in which possible combinations of predictive scoring components were progressively evaluated for face validity by experienced intensive care specialists. The local development of this triage tool to accompany the Admission, Discharge, and Triage Guidelines for Hong Kong (outlined above) was led by 10 senior ICU clinical specialists who are currently practising in ICUs in Hong Kong. The participants included at least one representative of each of Hong Kong's hospital cluster regions. Additionally, each participant routinely performs triage as a consequence of chronic ICU bed resource limitations. An initial meeting was held online, at which all key issues were discussed, and a draft document of the consensus view prepared by one author (GMJ). After circulation, several disagreements were documented. These were resolved by online voting, with a majority vote used to resolve persistent disagreement. Three rounds of online voting resulted in a finalised and universally supported document. A decision was made to respect and use the principles laid down in the pre-existing triage framework but to provide further detailed clinical guidance to frontline ICU doctors in Hong Kong regarding COVID-19. The starting point was to adapt and modify a recently published COVID-19 triage prioritisation tool developed by an international expert group. ${ }^{14}$ This tool took the form of a decision-making algorithm based on established ICU prognostic scoring systems that could inform bedside decision making in the event that ICU bed capacity becomes overwhelmed by patients with COVID-19. Therefore, the specific aim of the triage tool is to provide explicit and uniform guidance to all frontline doctors charged with the responsibility of triaging ICU admissions. This guidance should improve the objectivity and consistency of triage decision making across Hong Kong. The triage tool was designed to be easily understood, rapidly implemented, and of high utility. A list of the major considerations addressed to achieve this goal is provided in Table 2 .

\section{Results}

The first inclusion and exclusion criteria chosen for the triage tool (Fig 2) were those that, when answered, would rapidly finalise the decision without the need to proceed further, and thus prevent excessive use of valuable medical team time. Therefore, we created clear clinical exclusion criteria. Patients who are too ill to gain substantial incremental benefits from ICU care and those who refuse ICU admission on the basis of the perceived benefits and burdens of ICU care are excluded.

The explicit exclusion criteria are the same ones generally used in Hong Kong ICUs under normal circumstances. We chose general rather than specific diagnoses, as has been proposed previously, as specific diagnoses require the construction of long (but not exhaustive) lists.

The inclusion criteria are also directly comparable with the major inclusion criteria for ICU admission during 'normal' conditions: they reflect the need to admit patients who require ICU care to derive a survival benefit. Thus, patients who are 'too well' (ie, they can be reasonably treated in the ward) are excluded.

When a patient meets the inclusion criteria and does not meet any exclusion criteria, they become a potential ICU admission, and further priority is determined. Patients are subsequently chosen for admission based on their priority rank, ranging from 1 -high priority to 3 -low priority. A prioritisation score was developed by including variables that

TABLE 2. Key considerations in the development of the triage tool

1. Provision of support for immediate and rapid decision making that is needed at the bedside during an overwhelming pandemic and that will enhance consistency of decision making across different units

2. Provision of a triage tool that is simple and easy to understand and use

3. Provision of a tool that is flexible and adaptable to local practice, resource availability, and the changing severity of the outbreak

4. Provision of a tool that adheres to the existing ethical and functional principles of the Admission, Discharge, and Triage Guidelines

5. Individual triage decisions should ultimately be made or supervised by a senior ICU doctor based on the guidance provided by the triage prioritisation tool.

6. Patients with and without COVID-19 should be considered for priority on an equal basis.

7. The principles of triage implementation during the COVID-19 surge, and the contents of the triage tool, should be communicated to all relevant stakeholders, and if necessary, changes should be made on the basis of relevant feedback.

Abbreviations: COVID-19 = coronavirus disease 2019; ICU = intensive care unit 


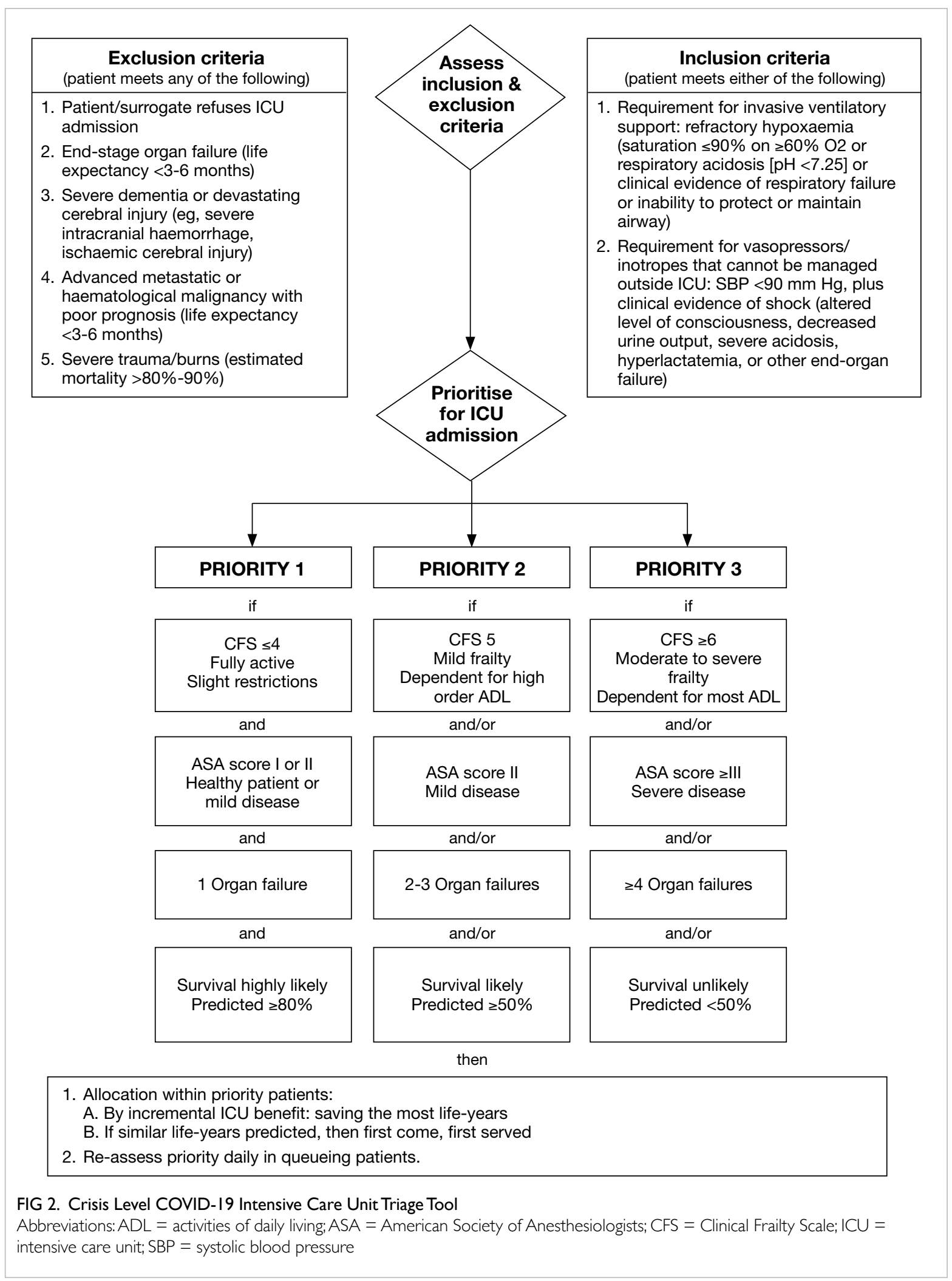

predict short-medium-term mortality (3-6 months) score $^{17}$ to assess co-morbidity (Table 3 ), and last, the in the first instance and are the most compatible with clinical assessment of the number of current organ the principle of 'quick and clear' decision making. system failures (OSF), that has previously been well The clinical frailty scale (CFS) [Fig 3], ${ }^{16}$ a modified established as an indicator to assist the prediction of American Society of Anesthesiologists (ASA) mortality. 
Outcome prognostication by the senior supervising ICU doctor is largely dependent on knowledge of the factors associated with poor outcomes and clinical experience. Although key relevant factors are captured by the tool, because COVID-19 is a new condition, we provide a table summarising mortality risk factors in patients with COVID-19 who are admitted to ICU to further aid prognostication (Table 4). ${ }^{18-20}$ The data were adapted from countries with ICU practices that are considered generally similar to those in Hong Kong.

Finally, it has been previously recommended that time-limited trials may be adopted at the time of admission. ${ }^{21}$ A time-limited trial establishes an agreement between the healthcare team and the patient/surrogate to apply necessary intensive care treatment for a pre-determined period of time. The ICU team keeps the family informed of patient progress, and when the pre-agreed time limit is reached, life support therapies are either continued if the patient has responded positively or withdrawn if therapy is failing. Setting an appropriate time period for the trial requires great care, ${ }^{22}$ and in the setting of COVID-19, care should be taken to allow sufficient time for the patient to respond to therapy. The median number of days of mechanical ventilation and the length of stay have been reported for patients with COVID-19 (10 days, and 9 to 12 days respectively), whose ICU stays are longer than those of patients with other viral pneumonias. ${ }^{18,19}$

The existing triage framework has been circulated for comment and feedback from relevant clinical specialty leadership groups in the Hospital Authority. Further, the current accompanying tool has been reviewed by the ad-hoc Hospital Authority Clinical Ethics Committee Core Group and finalised after incorporating relevant suggestions for change. After implementation, the triage working group will review the need for adjustment and updating of the guidelines according to local circumstances.

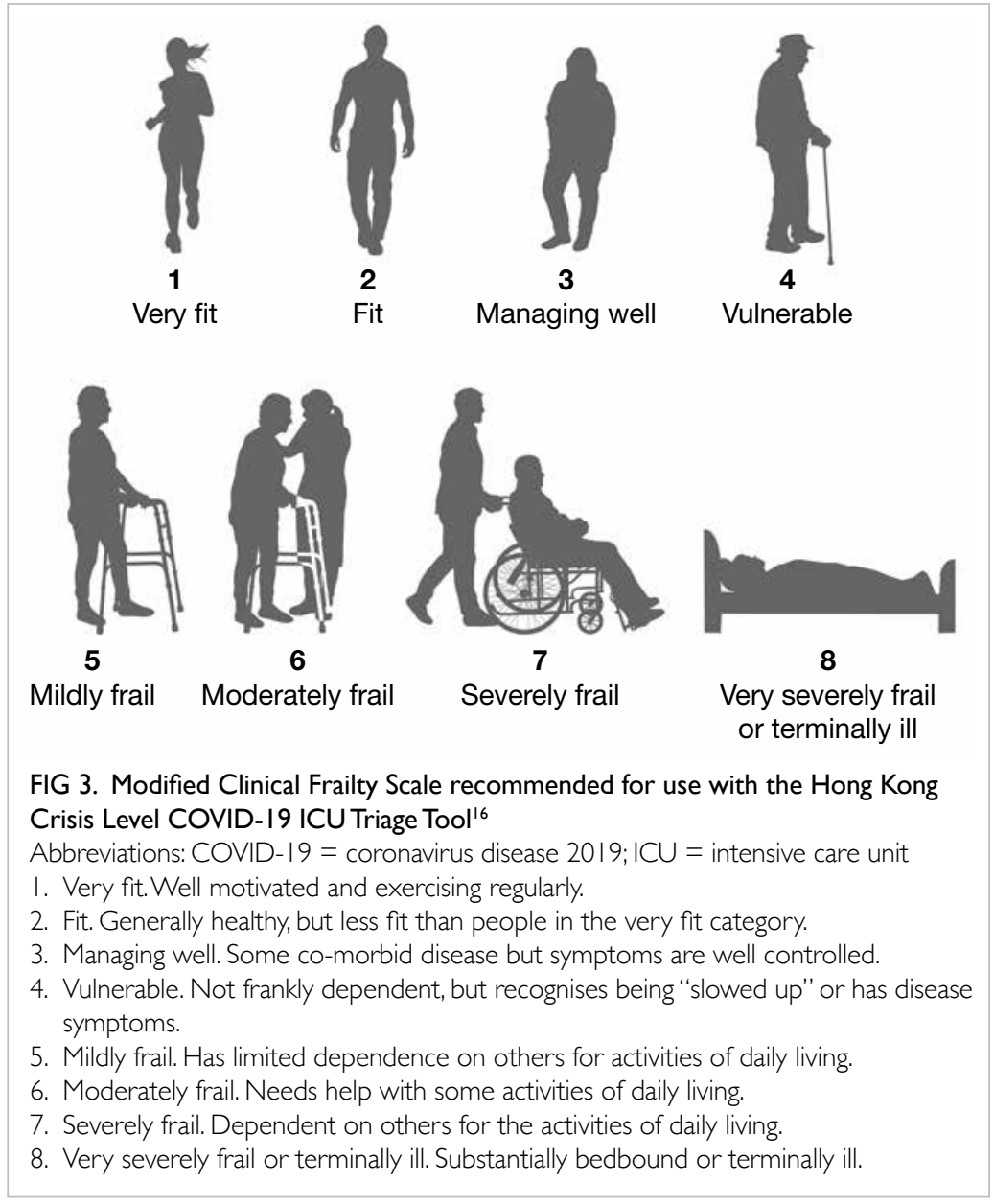

\section{Discussion}

The conceptual algorithm recommended herein broadly follows the existing recommended framework for individual triage decisions in that the inclusion criteria are based on a low likelihood of survival without ICU care $(5 \%-10 \%$ or less), if met. Priorities for admission can then be allocated

TABLE 3. Modified American Society of Anesthesiologists score for use with the Hong Kong Crisis Level COVID-I9 Intensive Care Unit Triage Tool ${ }^{17}$

\begin{tabular}{lll}
\hline Class & Description & Example \\
\hline I & The patient is previously healthy and fit & Normal effort tolerance for age \\
II & The patient has mild systemic controlled disease & $\begin{array}{l}\text { No substantive functional limitations: well-controlled diabetes mellitus or } \\
\text { hypertension, mild lung disease, effort tolerance } \geq 1-2 \text { flights of stairs }\end{array}$ \\
III & $\begin{array}{l}\text { The patient has severe but not incapacitating } \\
\text { systemic disease }\end{array}$ & $\begin{array}{l}\text { Substantive functional limitations: poorly controlled diabetes mellitus or hypertension, } \\
\text { COPD, moderate reduction of ejection fraction, effort tolerance <1-2 flights of stairs, } \\
\text { ESRD, history of MI, CVA, TIA, or CAD }\end{array}$ \\
IV & The patient has incapacitating systemic disease & $\begin{array}{l}\text { Severe functional limitations: ongoing cardiac ischaemia or severe valve dysfunction, } \\
\text { severe reduction of ejection fraction or effort tolerance restricted to short distances } \\
\text { on level ground }\end{array}$ \\
& Moribund and not expected to survive 24 hours & $\begin{array}{l}\text { Massive trauma or burn injury, severe intracranial bleed with mass effect, extensive } \\
\text { ischaemic bowel, significant cardiac pathology with severe reduction of ejection } \\
\text { fraction, or multiple organ/system failure }\end{array}$ \\
\hline
\end{tabular}

Abbreviations: CAD = coronary artery disease; COPD = chronic obstructive pulmonary disease; COVID-19= coronavirus disease 2019; CVA = cerebrovascular accident; ESRD = end-stage renal disease; $\mathrm{MI}=$ myocardial infarction; $\mathrm{TI} \mathrm{A}=$ transient ischaemic attack 
TABLE 4. Factors associated with increased mortality in critically ill patients admitted to ICU and reported estimates of risk ${ }^{18-20}$

\begin{tabular}{|c|c|c|c|}
\hline \multirow[t]{2}{*}{ Variable } & \multirow[t]{2}{*}{ Category (description) } & \multicolumn{2}{|c|}{ Multivariable } \\
\hline & & HR (95\% Cl) & OR $(95 \% \mathrm{Cl})$ \\
\hline Age in years & 10-Year increments & $1.75(1.60-1.92)$ & - \\
\hline Sex & Male vs female & $1.57(1.31-1.88)$ & - \\
\hline Hypercholesterolaemia & Present vs not present & $1.25(1.02-1.52)$ & - \\
\hline Type 2 diabetes & Present vs not present & $1.18(1.01-1.39)$ & - \\
\hline COPD & Present vs not present & $1.68(1.28-2.19)$ & - \\
\hline COPD or ILD & Present vs not present & $2.94(1.48-5.84)$ & - \\
\hline $\mathrm{FiO}_{2}$ at admission & $10 \%$ Increments & $1.14(1.10-1.19)$ & - \\
\hline Coronary artery disease & Present vs not present & - & $1.47(1.07-2.02)$ \\
\hline Active cancer & Present vs not present & - & $2.15(1.35-3.43)$ \\
\hline Coagulopathy & SOFA score component $\geq 2$ vs $<2$ & - & $1.64(1.00-2.69)$ \\
\hline Liver dysfunction & SOFA score component $\geq 2$ vs $<2$ & - & $2.61(1.30-5.25)$ \\
\hline Renal dysfunction & SOFA score component 1 -4 vs 0 & - & $1.56-2.43(1.20-4.05)$ \\
\hline Chronic kidney disease & Present vs not present & $1.76(1.08-2.86)$ & - \\
\hline $\mathrm{BMI} \geq 40$ & Present vs not present & - & $1.51(1.01-2.25)$ \\
\hline
\end{tabular}

Abbreviations: $95 \% \mathrm{Cl}=95 \%$ confidence interval; $\mathrm{BMI}=$ body mass index; $\mathrm{COPD}=$ chronic obstructive pulmonary disease; $\mathrm{HR}=$ hazard ratio; ICU = intensive care unit; ILD = interstitial lung disease; OR = odds ratio; SOFA = Sequential Organ Failure Assessment

on the basis of agreed-upon criterion thresholds for survival, as established in the accompanying triage tool and adjusted for Hong Kong's circumstances at a specific time. Thus, an incremental benefit of at least $40 \%$ to $45 \%$ would be required to meet the criteria for priority level 3, but one of at least $70 \%$ to $75 \%$ would be required to meet the criteria for priority level 1. Admission would depend on available resources after safe maximisation of surge capacity and the number of patients queuing for admission (eg, stricter incremental benefit thresholds may be required during the peak of the pandemic, and less strict thresholds may be implemented at the beginning and towards the end). The use of predicted incremental benefit for decision making has been previously endorsed by expert consensus groups when triage is required, both under outbreak and non-outbreak conditions. ${ }^{1,2,14}$

The CFS, which has nine variables, was chosen as the appropriate general health performance metric, as it meets local practice requirements: familiarity, ease of use, and having been validated as a predictor of short- and medium-term ICU outcomes. ${ }^{23-26}$ The well-established ASA score was chosen for modification to guide assessment of comorbidities, as its descriptions are clear, concise, and logically presented (ASA 2019). ${ }^{17}$ Further, the relationship between increasing OSF scores and higher mortality is well established..$^{27,28} \mathrm{~A}$ simple bedside clinical assessment of organ failure to decide the number of OSF is recommended, rather than attempting to determine the SOFA (Sequential Organ Failure Assessment) score, which requires additional calculations from clinical variables, assessment of missing variables, and then further prioritisation. ${ }^{29,30}$ We suggest using a clinical judgement for assessing end-stage organ failure of the noted organs (eg, brain, heart, lungs). However, individual units may choose to use the SOFA score if its calculation is considered achievable under local circumstances.

After deliberating at length, the group concluded that the indicative mortalities of the three chosen variables for determining the priority scores (general well-being [CFS], co-morbidities [ASA], and number of OSF) are such that in combination they are likely to correspond to the subjective predicted outcomes and survival percentages noted at the bottom of the notation for each priority score. The noted predicted survival percentages were calibrated with the recommendations of previous consensus expert groups, one who decided to define 'a minimal acceptable incremental ICU benefit' in a resource-limited setting as a $15 \%$ to $25 \%$ difference in mortality, ${ }^{10}$ and the second who adjusted this difference to be substantially larger (50\%) to account for the increased pressure anticipated in an outbreak setting. ${ }^{14}$ The use of the tool to guide the clinical estimation of likely benefits (outcome of ICU admission compared with outcome expected if the patient remained on the ward/other care area) is necessary for prioritisation of patients who will benefit most from ICU treatment. Nevertheless, because individual patients may have overriding characteristics not captured by the individual or combined scores, the final decision regarding likely incremental benefit and subsequent prioritisation 
should be made by the senior supervising triage doctor.

If there is more than one patient judged to be within the same priority group, and there is anticipated queuing for the remaining available beds, further prioritisation by incremental ICU benefit, such as saving the most life-years (evaluating mortality from both acute and chronic disorders) should be considered. If a tie for ICU admission candidates remains after these progressive steps, we recommend that admission be determined by the first-come, first-served principle.

Because of the complexity of the decisionmaking process and the multiple factors that require careful consideration, final decisions are best made by an experienced ICU doctor. However, should uncontrollable circumstances dictate that decisions need be made by a more junior colleague, the tool can still provide assistance to guide and enhance consistent and justifiable decision making. To prepare for this possibility, preparatory education should be provided to more junior colleagues regarding triage decision making to facilitate appropriate interpretation of this tool.

This tool specifically addresses the triage of patients for ICU admission. However, when available ICU resources are overwhelmed, enhanced levels of care within the ward or available high care areas should be used for the treatment of cases denied ICU admission. This could optimise patient outcomes within the constraints of available alternatives. In this regard, both invasive and non-invasive mechanical ventilation is routine practice in the wards and highcare areas of many hospitals in Hong Kong. This fact can potentially be harnessed for the treatment of COVID-19 cases. Patients denied ICU admission on the basis of triage should be preferentially considered for diversion to such resources. Hospitallevel coordination and close liaison between hospital facilities management and those who manage ICU resources is required to facilitate the appropriate use of all potentially available resources. ${ }^{31}$ Although this tool is designed specifically to guide ICU admission triage decisions, other users may consider using the priority assigned by the ICU triage officer to a refused case to allocate the patient to an appropriate next level of care.

Many bedside operational factors are part of the triage process but are not specifically embedded in this crisis tool. Nevertheless, they are substantially addressed in the current Admission, Discharge, and Triage Guidelines, of which the COVID-19 Crisis Triage Tool is an extension. These include the need for clear, empathic communication with patients and surrogates and the implementation of the appropriate best care plan, including palliation of symptoms when appropriate, to patients refused ICU admission. Clear and transparent communication with referring medical teams, mechanisms for audit and oversight, and channels for feedback and reassessment are also required.

Important limitations must be acknowledged. The current guideline is based on the consensus of experienced Hong Kong clinicians with a history of performing bedside triage and not high-level, published medical evidence. Although the prognostic systems chosen have been well demonstrated to align with survival prognosis and functional outcomes, prognostic uncertainty in intensive care cannot be overcome by a single scoring system. All prognostic scoring systems, including the $\mathrm{CFS},{ }^{32}$ have limitations, and for this reason, the simultaneous use of multiple scoring methods, as used in this tool, has been recommended..$^{15}$

\section{Conclusion}

The referral of a patient for ICU care triggers a complex triage (prioritisation) decision that must be made when ICU beds are limited. It is expected that this triage tool, in the form of a detailed decision aid algorithm, should increase objectivity and transparency in triage decision making and help to enhance consistency between doctors both within and across ICUs in Hong Kong. However, this tool is an aid rather than a complete substitute for the carefully considered judgement of an experienced intensive care clinician.

\section{Author contributions}

Concept or design: All authors.

Acquisition of data: All authors.

Analysis or interpretation of data: All authors.

Drafting of the manuscript: GM Joynt.

Critical revision of the manuscript for important intellectual content: All authors.

All authors had full access to the data, contributed to the study, approved the final version for publication, and take responsibility for its accuracy and integrity.

\section{Conflicts of interest}

All authors have disclosed no conflicts of interest.

\section{Funding/support}

This research received no specific grant from any funding agency in the public, commercial, or not-for-profit sectors.

\section{References}

1. Christian MD, Sprung CL, King MA, et al. Triage: care of the critically ill and injured during pandemics and disasters: CHEST consensus statement. Chest 2014;146(4 Suppl):e61S-74S.

2. Joynt GM, Gopalan DP, Argent AA, et al. The Critical Care Society of Southern Africa Consensus Statement on ICU Triage and Rationing (ConICTri). S Afr Med J 2019;109:613-29.

3. Ling L, Ho CM, Ng PY, et al. Characteristics and outcomes 
of patients admitted to adult intensive care units in Hong Kong: a population retrospective cohort study from 2008 to 2018. J Intensive Care 2021;9:2.

4. Phua J, Faruq MO, Kulkarni AP, et al. Critical care bed capacity in Asian countries and regions. Crit Care Med 2020;48:654-62.

5. Murthy S, Wunsch $\mathrm{H}$. Clinical review: international comparisons in critical care-lessons learned. Crit Care 2012;16:218.

6. Joynt GM, Gomersall CD, Tan P, Lee A, Cheng CA, Wong EL. Prospective evaluation of patients refused admission to an intensive care unit: triage, futility and outcome. Intensive Care Med 2001;27:1459-65.

7. Shum HP, Chan KC, Lau CW, Leung AK, Chan KW, Yan WW. Triage decisions and outcomes for patients with Triage Priority 3 on the Society of Critical Care Medicine scale. Crit Care Resusc 2010;12:42-9.

8. Nates JL, Nunnally M, Kleinpell R, et al. ICU Admission, Discharge, and Triage Guidelines: a framework to enhance clinical operations, development of institutional policies, and further research. Crit Care Med 2016;44:1553-602.

9. Blanch L, Abillama FF, Amin P, et al. Triage decisions for ICU admission: report from the task force of the World Federation of Societies of Intensive and Critical Care Medicine. J Crit Care 2016;36:301-5.

10. Joynt GM, Gopalan DP, Argent AA, et al. The Critical Care Society of Southern Africa Consensus Guideline on ICU Triage and Rationing (ConICTri). S Afr Med J 2019;109:630-62.

11. Legislative Council, HKSAR Government. Report of the Select Committee to inquiry into the handling of the severe acute respiratory syndrome outbreak by the Government and the Hospital Authority. 2004. Available from: https:// www.legco.gov.hk/yr03-04/english/sc/sc_sars/reports/ sars_rpt.htm. Accessed 15 Sep 2020.

12. Gomersall CD, Tai DY, Loo S, et al. Expanding ICU facilities in an epidemic: recommendations based on experience from the SARS epidemic in Hong Kong and Singapore. Intensive Care Med 2006;32:1004-13.

13. Guidet B, Hejblum G, Joynt G. Triage: what can we do to improve our practice? Intensive Care Med 2013;39:2044-6.

14. Sprung CL, Joynt GM, Christian MD, Truog RD, Rello J, Nates JL. Adult ICU triage during the coronavirus disease 2019 pandemic: who will live and who will die? Recommendations to improve survival. Crit Care Med 2020;48:1196-202.

15. Flaatten H, Beil M, Guidet B. Prognostication in older ICU patients: mission impossible? Br J Anaesth 2020;125:655-7.

16. Rockwood K, Song X, MacKnight C, et al. A global clinical measure of fitness and frailty in elderly people. CMAJ 2005;173:489-95.

17. American Society of Anaesthesiologists. ASA Physical Status Classification System. 2019. Available from: https:// www.asahq.org/standards-and-guidelines/asa-physicalstatus-classification-system. Accessed 16 Sep 2020.

18. Gupta S, Hayek SS, Wang W, et al. Factors associated with death in critically ill patients with coronavirus disease 2019 in the US. JAMA Intern Med 2020;180:1-12.

19. Grasselli G, Greco M, Zanella A, et al. Risk factors associated with mortality among patients with COVID-19 in intensive care units in Lombardy, Italy. JAMA Intern Med 2020;180:1345-55.

20. Cummings MJ, Baldwin MR, Abrams D, et al. Epidemiology, clinical course, and outcomes of critically ill adults with COVID-19 in New York City: a prospective cohort study. Lancet 2020;395:1763-70.

21. Quill TE, Holloway R. Time-limited trials near the end of life. JAMA 2011;306:1483-4.

22. Vink EE, Azoulay E, Caplan A, Kompanje EJ, Bakker J. Time-limited trial of intensive care treatment: an overview of current literature. Intensive Care Med 2018;44:1369-77.

23. Bagshaw M, Majumdar SR, Rolfson DB, Ibrahim Q, McDermid RC, Stelfox HT. A prospective multicenter cohort study of frailty in younger critically ill patients. Crit Care 2016;20:175.

24. Bagshaw SM, Stelfox HT, McDermid RC, et al. Association between frailty and short- and long-term outcomes among critically ill patients: a multicentre prospective cohort study. CMAJ 2014;186:E95-102.

25. Brummel NE, Bell SP, Girard TD, et al. Frailty and subsequent disability and mortality among patients with critical illness. Am J Respir Crit Care Med 2017;196:64-72.

26. Flaatten H, De Lange DW, Morandi A, et al. The impact of frailty on ICU and 30-day mortality and the level of care in very elderly patients ( $\geq 80$ years). Intensive Care Med 2017;43:1820-8.

27. Knaus WA, Draper EA, Wagner DP, Zimmerman JE. Prognosis in acute organ-system failure. Ann Surg 1985;202:685-93.

28. Peres Bota D, Melot C, Lopes Ferreira F, Nguyen Ba V, Vincent JL. The Multiple Organ Dysfunction Score (MODS) versus the Sequential Organ Failure Assessment (SOFA) score in outcome prediction. Intensive Care Med 2002;28:1619-24.

29. Vincent JL, Moreno R, Takala J, et al. The SOFA (Sepsisrelated Organ Failure Assessment) score to describe organ dysfunction/failure. On behalf of the Working Group on Sepsis-Related Problems of the European Society of Intensive Care Medicine. Intensive Care Med 1996;22:70710.

30. Ferreira FL, Bota DP, Bross A, Mélot C, Vincent JL. Serial evaluation of the SOFA score to predict outcome in critically ill patients. JAMA 2001;286:1754-8.

31. Joynt GM, Loo S, Taylor BL, et al. Chapter 3. Coordination and collaboration with interface units. Recommendations and standard operating procedures for intensive care unit and hospital preparations for an influenza epidemic or mass disaster. Intensive Care Med 2010;36(Suppl 1):S2131.

32. Darvall JN, Bellomo R, Bailey M, et al. Frailty and outcomes from pneumonia in critical illness: a population-based cohort study. Br J Anaesth 2020;125:730-8. 